\title{
Cálculo y análisis de la resiliencia de los departamentos de Colombia*
}

\section{Calculation and analysis of the resilience of the departments of Colombia}

\author{
Martha Yánez-Contreras ${ }^{1}$ \\ Universidad de Cartagena, Cartagena, Colombia \\ myanezc@unicartagena.edu.co \\ https://orcid.org/0000-0003-0559-5835 \\ Jorge Martelo-Amaya ${ }^{2}$ \\ Universidad de Cartagena, Cartagena, Colombia \\ j220493@gmail.com \\ https://orcid.org/0000-0001-8107-9790 \\ Haroldo Rodríguez-Páez ${ }^{3}$ \\ Universidad de Cartagena, Cartagena, Colombia \\ hrodriguezpl@unicartagena.edu.co \\ https://orcid.org/0000-0001-7304-7609
}

Recibido: 14-11-19

Aprobado: 10-06-20

* Este artículo es resultado del proyecto de investigación "Resiliencia urbana en Colombia", desarrollado por el grupo de investigación, de la Universidad de Cartagena, Economía y Gestión del medio ambiente; el cual fue favorecido y financiado con recursos de la octava convocatoria de proyectos de investigación realizada por la vicerrectoría de investigaciones de la Universidad de Cartagena.

1 Magister en Economía del Medio Ambiente.

2 Especialista en Estadística.

3 Economista. 


\title{
Resumen
}

En este artículo se analiza y compara la resiliencia de los departamentos de Colombia para mitigar los efectos de eventos adversos. Para esto se construyó un índice compuesto de resiliencia, utilizando variables como la diversidad de suelos, la diversidad de empresas, el índice de desempeño integral y la capacidad de carga sobre el ecosistema. Los resultados obtenidos evidencian gran disparidad entre los niveles de resiliencia de los entes territoriales estudiados, al tiempo que refuerzan la concepción holística de la resiliencia. De manera que los cálculos obtenidos para el índice son explicados por el desempeño integral de sus componentes.

Palabras clave: resiliencia; desarrollo sostenible; capital social.

Clasificación JEL: Q01, Q50, Q57, R14.

\begin{abstract}
In this paper the resilience of the departments of Colombia in order to mitigate the effect of adverse events will be both analyzed and compared. To accomplish this, an index was build composed by resilience, using variables such as the soil diversity, enterprises variety, the integral performance index and the carrying capacity on the ecosystem. The results show a great disparity among the levels of resilience within the territorial subjects studied while also reinforcing the holistic conception of resilience. Thus, the calculations obtained for the index are explained through the integral performance of their components.
\end{abstract}

Keywords: resilience; sustainable development; share capital.

JEL Classification: Q01, Q50, Q57, R14. 


\section{Introducción}

La resiliencia es un concepto propuesto inicialmente en el campo de la ecología por Holling (1973), el cual ha sido usado en distintas áreas de estudios como son la psicología, la física de materiales, la ingeniería, la sociología, la economía, entre otros (Brand y Jax, 2007). Por consiguiente, en las discusiones generadas sobre los factores que posibilitan el desarrollo territorial, la resiliencia se ha incorporado como una variable cuyos efectos pueden ser cruciales al momento de evaluar el impacto que tiene un evento adverso sobre un territorio. Este es un concepto muy relacionado con la capacidad de adaptación, la vulnerabilidad y la sostenibilidad de los sistemas. Por lo tanto, para la promoción de la resiliencia deben existir sistemas de gobiernos robustos que puedan hacer frente a los cambios sociales, económicos y ambientales que se presenten (Campos-García, 2013).

$\mathrm{Al}$ ser un concepto multidimensional, disponer de información sobre la resiliencia territorial, en tanto permite conocer cómo el territorio respondería ante eventos adversos, generaría una ventaja a la hora de tomar las decisiones orientadas a mitigar los efectos producidos por una presión externa. Esto ha dado paso a iniciativas como el Marco de Acción de Hyogo: una herramienta a la que se acogieron los países miembros de las Naciones Unidas, y que tiene como objetivo aumentar la resiliencia de las naciones y comunidades ante los desastres, buscando así reducir las pérdidas que estos ocasionan (Organización de las Naciones Unidas, 2005).

En este mismo sentido, estudios realizados por la CEPAL (2018) destacan la importancia de potenciar la resiliencia de las ciudades y demás territorios, teniendo en cuenta las agendas del cambio climático ya que, en el contexto actual, es importante estar preparados ante cualquier eventualidad. De allí la importancia de asegurar la incorporación de políticas publicas de gestión de riesgos a los planes estratégicos de los gobiernos.

Para el desarrollo de este análisis, se construyó un índice compuesto de resiliencia, utilizando la metodología propuesta por Suarez-Casado (2012) que, a través de cuatro indicadores simples, representa la dimensión económica, social y ambiental de los departamentos estudiados. A partir de este índice se han identificado los departamentos más resilientes en Colombia, es decir, cuáles son capaces de afrontar de mejor manera un estrés externo. Este documento se encuentra estructurado de la siguiente forma: inicialmente, se presenta una revisión de la literatura existente; luego, se describe la metodología utilizada para la construcción del índice compuesto de resiliencia; para, más adelante, presentar y discutir los resultados alcanzados; finalmente, se presentan las conclusiones obtenidas.

\section{Resiliencia territorial}

La estabilidad de los sistemas complejos ha adquirido una creciente importancia, a partir de la década de los 70 , convirtiéndose en objeto de análisis e investigaciones en diversos campos de estudio. Es Holling (1973) quien emplea por primera vez el término de resiliencia, definiéndolo como la capacidad de un ecosistema de absorber perturbaciones sin alterar significativamente sus características. De esta manera, vuelve a su estado inicial cuando la perturbación finaliza. Posterior a una perturbación, la característica resiliente del sistema genera un proceso de recuperación que permite llegar al estado inicial previo al shock, como explican Pimm (1984), Gunderson (2010), Martin-Breen y Anderies (2011); o, tal como ocurre frecuentemente en los ecosistemas, que se alcance un nuevo balance, producto de la imposibilidad de retornar al equilibrio inicial (Holling, 1996; Magis, 2010).

Una de las principales divisiones que se ha generado, a partir de este concepto, es la forma como se aborda. En algunos campos, la resiliencia se muestra como un resultado, es decir, un lugar es resiliente hasta el punto en que se recupera de la fluctuación; mientras que en otras áreas es considerada una capacidad. En este caso, un lugar se considera resiliente hasta el punto en que tiene las condiciones para recuperarse potencialmente (Foster, 2012; Rizzi et al., 
2018). La resiliencia, generalmente, ha sido considerada como un atributo global: la capacidad de recuperación del sistema de forma holística, como un todo. Sin embargo, en ocasiones, una perturbación puede no alterar las propiedades de todos sus elementos, es decir, el impacto de un fenómeno no es uniforme en todos sus componentes, indicando que los elementos individuales pueden tener características resilientes heterogéneas (Walker et al., 2004; Folke et al., 2010; Gunderson, 2010).

La aplicación del concepto de resiliencia, en diferentes áreas de las ciencias sociales, ha provocado la aparición de nuevos términos como la resiliencia urbana y la resiliencia territorial. Ambas derivaciones se han enfocado principalmente en sistemas como las ciudades y las regiones, las cuales son consideradas como espacios comunitarios caracterizados por el desarrollo y el uso de recursos comunes por parte de sus integrantes, para así perdurar ante las constantes fluctuaciones (Berkes y Ross, 2016).

En particular, al hablar de resiliencia territorial se hace referencia a la capacidad que tienen algunos territorios como ciudades, regiones, entes territoriales, áreas rurales, entre otros, de preparar, resistir, o adaptarse a situaciones adversas de tipo económico, social, político, ambiental, epidemiológico o tecnológico, que afectan los elementos que conforman el territorio, la población y sus actividades (Sánchez- Zamora et al., 2014; Berga, 2017).

Al respecto, Sánchez-Zamora et al. (2014), siguiendo a Hassink (2010), Pike et al. (2010) y Hamdouch et al. (2012), explican que la resiliencia territorial tiene dos conceptualizaciones. $\mathrm{La}$ primera hace referencia a la "resiliencia estática", que mide la capacidad del territorio para soportar presiones externas, manteniendo las bases de su desarrollo. Es decir, la resiliencia estática es la que permite al territorio volver a un estado de equilibrio. El segundo concepto es el de la "resiliencia dinámica", definida como la facultad permanente que posee un territorio de generar las competencias necesarias que le permitan adaptarse favorablemente a la dinámica de los entornos cambiantes. La característica principal de la resiliencia dinámica es la capacidad de adaptación y aprendizaje, en el largo plazo, ante los shocks externos.

$\mathrm{Al}$ abordar las características que hacen a un territorio resiliente, algunos autores como Bruneau et al. (2003), Foster (2012), Rizzi et al. (2018), Norris et al. (2008), Polèse (2010), Christopherson et al. (2010), coinciden en que los determinantes de corte económico y social, como la cualificación del recurso humano, la diversificación de la economía y la existencia de políticas orientadas al desarrollo social, son elementos muy importantes en la resiliencia de los territorios. De manera similar, para Labandeira et al. (2007), la principal característica que debe tener cualquier sistema que desee construir una resiliencia robusta es la diversidad. Característica que puede ser extendida a las ciudades, departamentos, regiones y demás entes territoriales, dado que cuentan con una gran cantidad de elementos interactuando entre sí.

\section{Capital social}

El concepto de capital social es definido por Putnam (1993) como las características de la organización social, tales como las redes, la confianza, y las normas que facilitan la cooperación y la búsqueda del beneficio mutuo. El capital social se encuentra estrechamente relacionado con la resiliencia, pues este es el elemento principal de la dimensión social que compone el concepto de la resiliencia territorial. Es decir, los territorios que poseen altos índices de capital social serán más resilientes, ya que competencias como el sentido de pertenencia, la educación, las redes sociales, la cultura y las buenas instituciones, fomentan el desarrollo de un territorio y, por tanto, tienen un impacto positivo en los niveles de resiliencia del mismo (Ledogar y Fleming, 2008).

En ese orden de ideas, autores como Méndez (2013), Walker et al. (2004), Méndez (2015) y Adgner (2000) también resaltan la importancia del capital social y la innovación como parte integral de un sistema resiliente, pues el capital social -representado en las redes que se forman entre los agentes económicos, la participación 
ciudadana en la toma de decisiones y en la elección de sus representantes- permite tener instituciones fuertes que, a su vez, ayudan al desarrollo económico y social del sistema. Así mismo, la innovación permite superar el estancamiento económico de un ente territorial, ayudando al mejoramiento de las empresas y, además, es un factor diferencial a la hora de superar los shocks externos que aflijan al sistema.

Lo anterior permite observar que la institucionalidad tiene un efecto de causalidad sobre el capital social, de tal manera que instituciones eficientes e íntegras, responsables de la implementación de políticas públicas, influencian positivamente en la creación de capital social (Rothstein y Stolle, 2002). Por ello, ante una agitación o perturbación, el sistema urbano produce un proceso inevitable de creación de capital social, el cual se fortalece a través de las instituciones (Dynes, 2002; Quarantelli, 2004).

\section{Evidencia empírica}

Son variadas las metodologías usadas para medir la resiliencia. Por ejemplo, Carpenter et al. (2001) aplican el modelo del ciclo adaptativo en un sistema de lagos, pues para ellos el ciclo adaptativo es la llave para comprender la resiliencia. Por su parte, Milman y Short (2008) desarrollaron un indicador de resiliencia para evaluar la capacidad de un sistema de agua urbano de mantener o mejorar el nivel actual y la calidad del acceso en los próximos 50 años. Para ello, tomaron como base el modelo presión-estado-respuesta. En otro caso, Sánchez-Zamora et al. (2014), a través del análisis envolvente de datos, estudiaron los niveles de resiliencia territorial del medio rural andaluz, en dos periodos de tiempo diferentes, relacionados por las épocas de crisis económicas, y teniendo en cuenta los tipos de capital (económico, humano, social, cultural y natural) como determinantes importantes de la capacidad de resiliencia de un territorio.

De manera similar, Cuadrado-Roura y Maroto (2016) han analizado la resiliencia territorial por regiones en España, después de la crisis financiera de 2008. Para ello, llevaron a cabo un análisis de la productividad y la especialización en las distintas regiones que conforman el territorio español. Los resultados mostraron que existe un desbalance entre las capacidades de recuperación de las regiones frente a la crisis económica: las regiones que previamente eran más ricas resultaron ser las más resilientes, debido a que se habían especializado y tenían alta productividad en industrias dinámicas como la de energía, manufacturas y servicios del mercado. El uso de los indicadores para analizar la resiliencia territorial ha sido frecuente. En ese sentido, Vincent (2007) ha desarrollado dos índices compuestos, a distintas escalas (localy nacional), para medir la capacidad de adaptación, usando cinco subíndices compuestos, ponderados según su representatividad. Estos subíndices son el bienestar económico y estabilidad (20\%); la estructura demográfica (20\%); la estabilidad institucional y bienestar (40\%); la interconectividad global (10\%); y la dependencia de los recursos naturales sensibles al estrés hídrico y la disponibilidad de agua (10\%).

Brooks et al. (2005) construyeron un indicador de vulnerabilidad y capacidad de adaptación al cambio climático con datos del nivel nacional y en un periodo de 10 años. Para la construcción del indicador, utilizaron variables proxis para representar las distintas categorías que lo componen, como lo son el PIB per cápita, el coeficiente Gini, la esperanza de vida al nacer, el control de la corrupción, la efectividad del gobierno, el porcentaje de tierra protegida, la tasa de cambio forestal, entre otras variables económicas, sociales y ambientales. Análogamente, Yohe y Tol (2002) construyeron un indicador que mide la capacidad de adaptación y la vulnerabilidad, teniendo en cuenta una serie de determinantes enmarcados por variables como el stock de capital humano, incluida la educación y seguridad personal; y el stock de capital social, incluida la definición de derechos de propiedad y la capacidad de los responsables de la toma de decisiones para gestionar la información. 


\section{Aspectos metodológicos}

En el desarrollo de esta investigación, se construyó un índice compuesto de resiliencia para 22 de los 32 departamentos de Colombia. Los departamentos estudiados son los utilizados por el Departamento Administrativo Nacional de Estadística (DANE), en la Encuesta Nacional Agropecuaria (ENA), del año 2012.

\section{1 Índice compuesto de resiliencia, datos y fuentes de información}

El índice compuesto de resiliencia está basado en el desarrollado por Suárez-Casado (2012). Se adaptaron las variables para el caso colombiano, reemplazando algunas como la agenda 21 española por el desempeño integral de los departamentos. Algo similar se realizó con la huella ecológica por departamentos, ya que en Colombia no ha sido estimada para estos entes territoriales, por lo que se efectuó un cálculo aproximado. El índice está desarrollado de la siguiente manera:

$$
\text { Resiliencia }=\frac{0,25 H(D \text { empresas })+0,25 H(D \text { usos del suelo })+0,5 D I}{\frac{H E}{S U P}}
$$

La variable $\boldsymbol{D}$ empresas corresponde a la diversidad de empresas, la cual se calcula dividendo la totalidad de las empresas que están en un departamento entre los grupos económicos en los que se separan las actividades. La información es tomada de la página web del Banco de Comercio Exterior de Colombia (BANCOLDEX, 2012), el cual a su vez toma la información de la Planilla Integrada de Liquidación de Aportes (PILA).

La variable $\boldsymbol{D}$ uso del suelo, que representa la diversidad de los suelos, es tomada de la Encuesta Nacional Agropecuaria (ENA), realizada por el DANE (2012b), la cual estima el uso de la tierra; el tamaño y distribución de los segmentos de muestreo; el área, producción y rendimiento de los principales cultivos transitorios y permanentes; el área en pastos, la producción de leche y el inventario pecuario.

La variable DI hace referencia al índice de desempeño integral, el cual se obtuvo dentro del panel municipal del Centro de Estudios sobre Desarrollo Económico (CEDE, 2012), en la sección de buen gobierno. El indicador de desempeño integral es calculado por el DNP y evalúa el desempeño de las entidades territoriales en cuanto a la eficacia en el cumplimiento de las metas de sus planes de desarrollo, la eficiencia en la provisión de los servicios básicos de educación, salud y agua potable, el desempeño fiscal y la capacidad administrativa. Para completar el índice de los departamentos, se promediaron los valores municipales y se obtuvo el índice departamental.

La variable $\boldsymbol{H E}$ hace referencia a la huella ecológica, la cual representa el área de tierra o mar, biológicamente productiva, requerida para satisfacer las actuales necesidades ambientales de la especie humana (Labandeira et al., 2007; Wackernalgel y Rees, 1996). Para calcularla, se usó la metodología propuesta por Loh y Wackernagel (2004), a partir de variables como el área de bosque, pastos, tierra de uso agrícola, área de pesca, emisiones de $\mathrm{CO} 2$ de combustibles y energía, representadas en hectáreas globales por persona. Los datos para su construcción fueron tomados de la ENA realizada por el DANE (2012b).

Para medir el componente de la energía, que hace parte del cálculo de la $\boldsymbol{H E}$, se tomaron los datos del Inventario Nacional de Gases de Efecto Invernadero, realizado por el IDEAM (2012). En este informe, se presentan las emisiones por sectores a nivel nacional y departamental, referidas a distintos gases como el $\mathrm{CO}_{2}, \mathrm{CH}_{4}, \mathrm{~N} 2 \mathrm{O}, \mathrm{HFC}$, SF6 y PFC, y generadas a través de las diferentes actividades económicas y humanas. Para su cálculo, se utilizaron las emisiones de $\mathrm{CO} 2 \mathrm{e}$ procedentes 
de los combustibles utilizados en el sector de transportes, las emisiones de $\mathrm{CO}_{2} \mathrm{e}^{4}$ del sector de la industria de la energía y las emisiones de $\mathrm{CO}_{2} \mathrm{e}$ procedentes de las actividades forestales. Estas tres actividades nos marcarían la aproximación de la huella ecológica energética por departamentos.

Luego de tener las anteriores variables se procedió a convertirlas a hectáreas. Las primeras cuatro, que son obtenidas de la ENA, ya se encuentran expresadas en esa unidad de medida, pero las últimas tres estaban expresadas en kilotoneladas 5 de Coze. Para convertir estas variables a hectáreas, se requirió conocer cuántas hectáreas de bosque absorben una cantidad $\mathbf{X}$ de gramos y/o toneladas de Co2, en un año, en Colombia. Dato que fue tomado del trabajo de Phillips et al. (2014) donde indica que, en el periodo de 1990-2013, los bosques en Colombia (59.134.663 de hectáreas) absorbieron, al año, entre 0,4 Pg $^{6}$ CO2e y 0,7 Pg CO2e.

Los datos obtenidos para las variables anteriores fueron divididos entre el tamaño de la población de cada departamento y, a partir de la sumatoria de las mismas, se obtuvo la huella ecológica por departamentos, representada en hectáreas por personas. Por último, al dividir la huella ecológica obtenida por la superficie de cada departamento, se generó la variable $\mathbf{H E / S U P}$, que hace referencia a la capacidad de carga, la cual es utilizada en el denominador de la fórmula para obtener el índice compuesto de resiliencia.

\subsection{Normalización de los datos para el desarrollo del índice compuesto de resiliencia}

Las variables $\boldsymbol{D}$ empresas y $\boldsymbol{D}$ uso del suelo permiten observar cómo están divididas las empresas y los suelos en los departamentos, pero para el cálculo del indicador propuesto es necesario medir la biodiversidad específica y, como explica Suarez-Casado (2012), siguiendo a Rueda (2011), la mejor manera de medir la

4 Coze hace referencia al dióxido de carbono equivalente (IDEAM, 2012).

5 Una kilotonelada equivale a mil toneladas (IDEAM, 2012).

6 Pg hace referencia a la unidad de medida petagramos que equivalen a 1000000000000000 gramos. diversidad de un ecosistema urbano, con las variables propuestas y los entes territoriales estudiados, es utilizando el índice de diversidad de Shannon.

Este índice está representado por:

$$
H=\sum_{i=1}^{n} P i \log (2) P i
$$

Donde:

$$
\mathrm{H}=\text { índice de diversidad de Shannon. }
$$

$\mathrm{Pi}=$ es la probabilidad de ocurrencia $\mathrm{o}$, en este caso, la proporción de individuos de la especie $i$ del total de la muestra $n$.

Este índice refleja la abundancia y la riqueza de las especies. En este caso, de las empresas y del uso de los suelos. Para el caso de la variable D empresas se utilizó la formula anterior y se clasificaron las empresas en 9 sectores económicos, teniendo resultados entre o como el valor mínimo de diversidad y 3 como el valor máximo de esta. Para la variable $\boldsymbol{D}$ uso del suelo sucede algo similar, se aplicó el índice de Shannon, se clasificó el uso del suelo en los cuatro sectores que vienen dados en la ENA y los valores de diversidad vienen dados como o el mínimo y 2 el máximo.

Por otro lado, la variable DI, que mide el desempeño integral de los gobiernos, en principio se encontraba en una escala de calificación promedio de o a 100 para los departamentos; para efectos de este trabajo, se convirtieron los datos, de forma que pudieran ser expresados en una proporción de 1 a 10.

Para la normalización de las tres variables anteriores se utilizó la siguiente formula:

$$
i=\frac{\text { valor real }- \text { valor mínimo }}{\text { valor máximo }- \text { valor mínimo }}
$$

A partir de ella, se normalizaron las variables $D$ empresas, D uso del suelo y DI, re escalando sus valores entre 0 y 1 , lo cual hizo posible agregarlas como un solo subíndice (Suarez-Casado, 2012). Con respecto a las ponderaciones, los valores fueron asignados dada la importancia de las dimensiones que conforman el entorno 
humano, la dimensión económica, social y ambiental.

Tanto la variable $\boldsymbol{D}$ empresas como la $\boldsymbol{D}$ uso del suelo representan la dimensión económica y cada una tiene una ponderación de 0,25 ; la variable $\boldsymbol{D I}$ representa la dimensión social a través del capital social, y tiene una ponderación del 0,50, dada su importancia en la capacidad de resiliencia. Por último, el denominador HE/SUP representa la dimensión ambiental, la cual es de suma importancia, ya que el medio ambiente es el que provee los recursos y el entorno en donde se desenvuelven y viven los seres humanos. Esta variable mide la carga o el impacto de los seres humanos en el ambiente y además no fue necesario normalizarla.

\section{Resultados}

\subsection{Estadísticas descriptivas de las variables}

La Figura 1 muestra cómo los departamentos de Antioquia, Cundinamarca, Santander y Valle del Cauca tienen el PIB más alto en relación con los demás departamentos. Además, son los únicos que superan el promedio del PIB entre los 22 departamentos analizados. Departamentos como Quindío, Sucre y la Guajira son los que tienen el PIB más bajo de la muestra, respectivamente. Los departamentos de Atlántico y Bolívar son los que mejores resultados muestran en la costa Caribe.

Teniendo en cuenta la Figura 2, para el año 2012 se aprecia que los departamentos con mayor renta per cápita son Casanare, Meta, Santander y Cundinamarca. El resultado de los dos primeros departamentos se explica por el bajo número de población que los conforma; pero cabe resaltar los resultados de Cundinamarca, Santander, Valle del Cauca y Antioquia, ya que son departamentos con altas tasas de población y presentan un alto nivel de renta -por encima del promedio de los departamentos estudiados- lo que muestra que son entes territoriales con un alto nivel de bienestar económico. Los departamentos de Nariño, Sucre, Magdalena y Córdoba, respectivamente, son los que tienen los niveles más bajos en su ingreso per cápita. También se puede observar que, a excepción del Cesar, ningún departamento de la costa Caribe supera el nivel de renta promedio per cápita nacional.

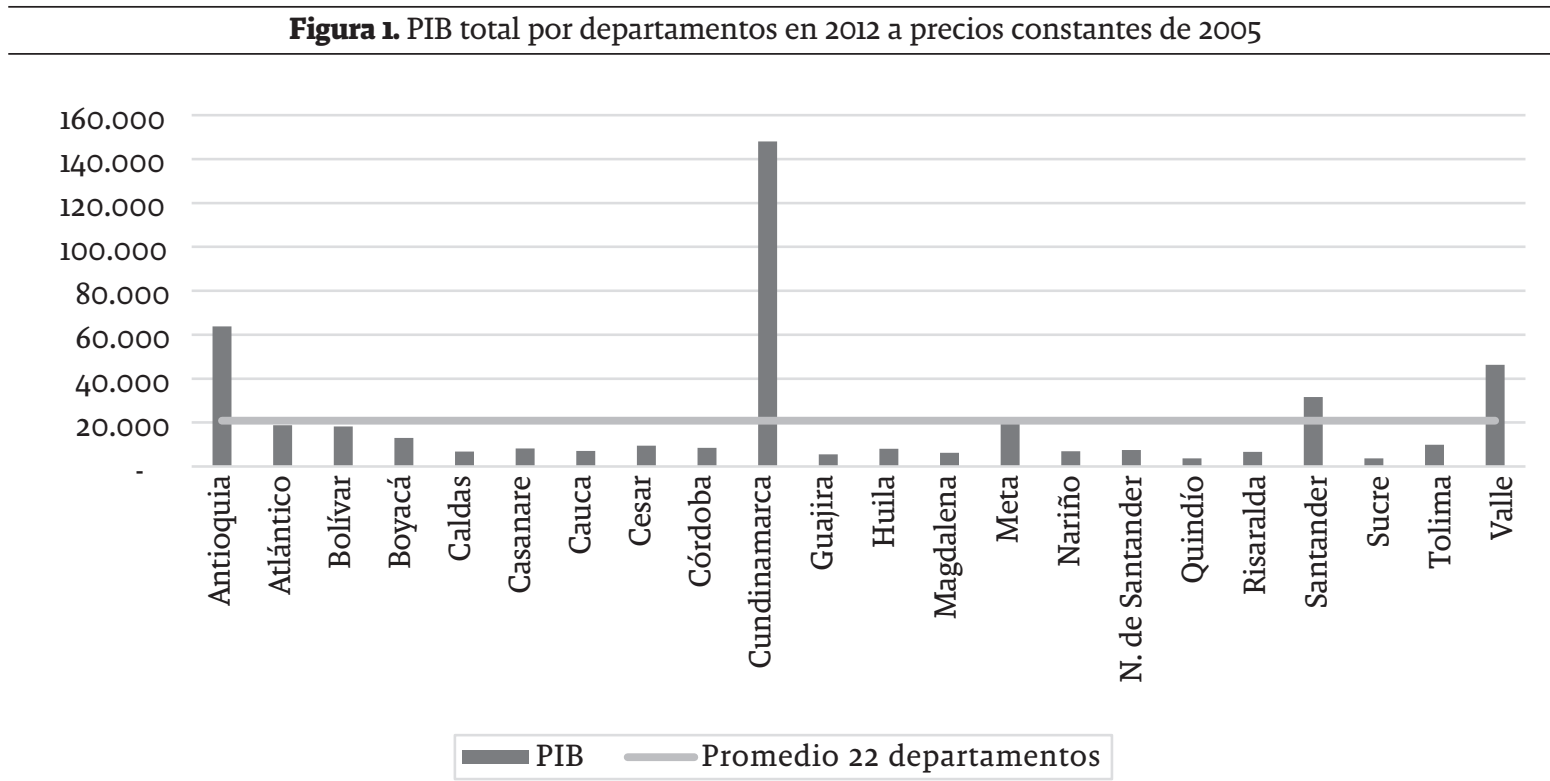

Nota: los resultados se encuentran en miles de millones de pesos.

Fuente: elaboración propia con datos de las cuentas departamentales del DANE (2012a). 
Figura 2. PIB per cápita por departamentos en 2012 a precios constantes de 2005

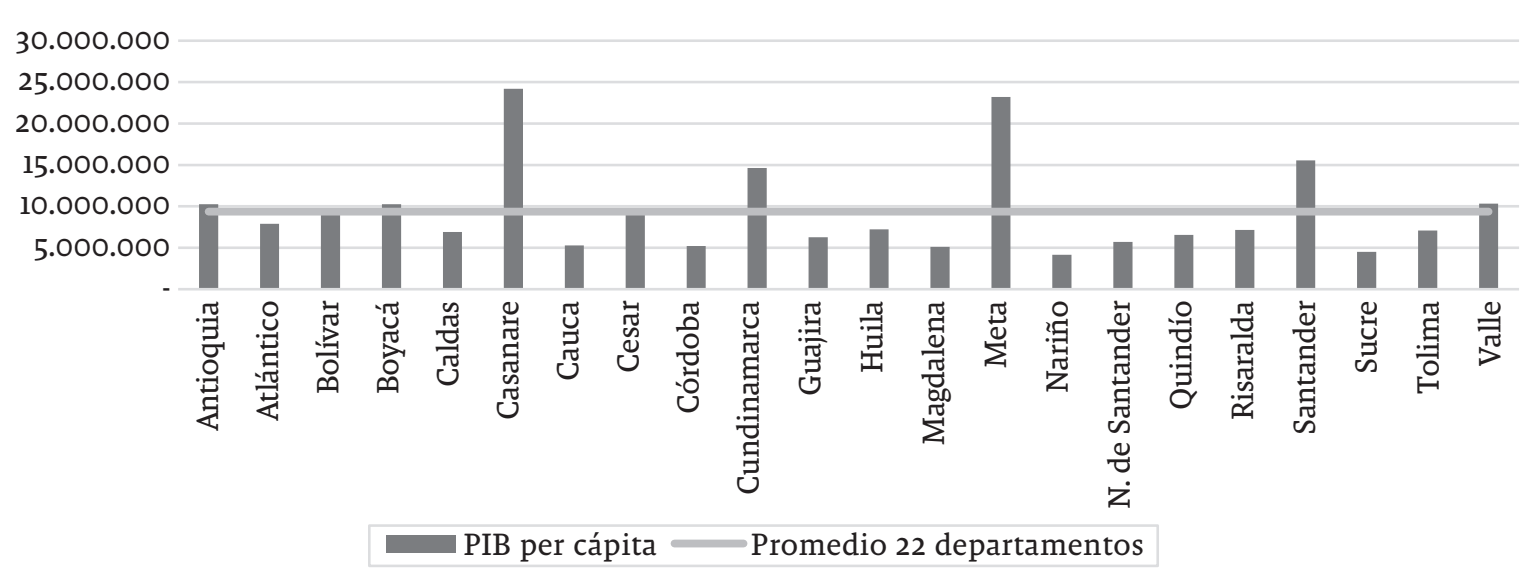

Nota: los datos se expresan en pesos.

Fuente: elaboración propia con datos de las cuentas departamentales del DANE (2012a).

\subsubsection{Uso del suelo}

En la Figura 3 se muestra la proporción de las actividades en las que se usan los suelos en los 22 departamentos estudiados. Se aprecia cómo, en promedio, los suelos son usados en su inmensa mayoría para actividades pecuarias como pueden ser la cría de ganado vacuno, porcino, avícola, la siembra de pastos, entre otras. También se puede observar que, en promedio, el uso de la tierra para actividades agrícolas y forestales es el mismo, y que el uso de la tierra para otros fines son las actividades de menos peso en la muestra.

Los departamentos de Risaralda y Quindío son los que más tierra utilizan para actividades del sector agrícola, a diferencia de la Guajira y el Meta, donde se destina menor cantidad de la tierra para esta actividad. Todo lo contrario sucede con el uso pecuario del suelo, ya que la Guajira, Meta y Casanare son los departamentos que más tierra orientan a ese uso, mientras que Risaralda y Quindío muestran menor grado de especialización en la actividad pecuaria. Cabe resaltar que la tierra utilizada en actividades pecuarias es superior a aquella usada en actividades agrícolas y bosques. Los departamentos que poseen mayor cantidad de hectáreas de bosques son los de Boyacá, Norte de Santander y Quindío; y aquellos con menor disposición de estos ecosistemas son los departamentos de la Guajira y Córdoba, respectivamente.

\subsubsection{Participación empresarial en los sectores económicos}

La Figura 4 muestra que, en promedio, la mayor concentración de las empresas, en los departamentos analizados, se encuentra desarrollando actividades del sector terciario. En efecto, las firmas de servicios financieros y empresariales representan un $39,2 \%$; los servicios sociales, un $27,8 \%$; y las firmas comerciales, un $19,2 \%$. Es decir, estas tres actividades acumulan el $86,3 \%$ del total de las empresas de los 22 departamentos estudiados.

Con respecto a las actividades agrícolas, los departamentos con mayor porcentaje de empresas son Cundinamarca, Meta y Casanare; por otra parte, los departamentos que menos empresas tienen, dentro de este sector, son los de Nariño, Atlántico, Bolívar, Norte de Santander y Guajira, con participaciones por debajo del $1 \%$. Respecto al comercio, la Guajira, Norte de Santander y Quindío son los entes territoriales con más empresas dentro de esta actividad; mientras que los de menos presencia empresarial son los departamentos de Boyacá, Casanare y Atlántico. En el sector de la construcción, los departamentos que más empresas registran son los de Casanare, Valle del Cauca y Meta, respectivamente; situación contraria se presenta en los departamentos de Sucre, Tolima y Magdalena, que tienen muy pocas firmas dedicadas a esta actividad. 
Figura 3. Uso del suelo por departamentos en 2012

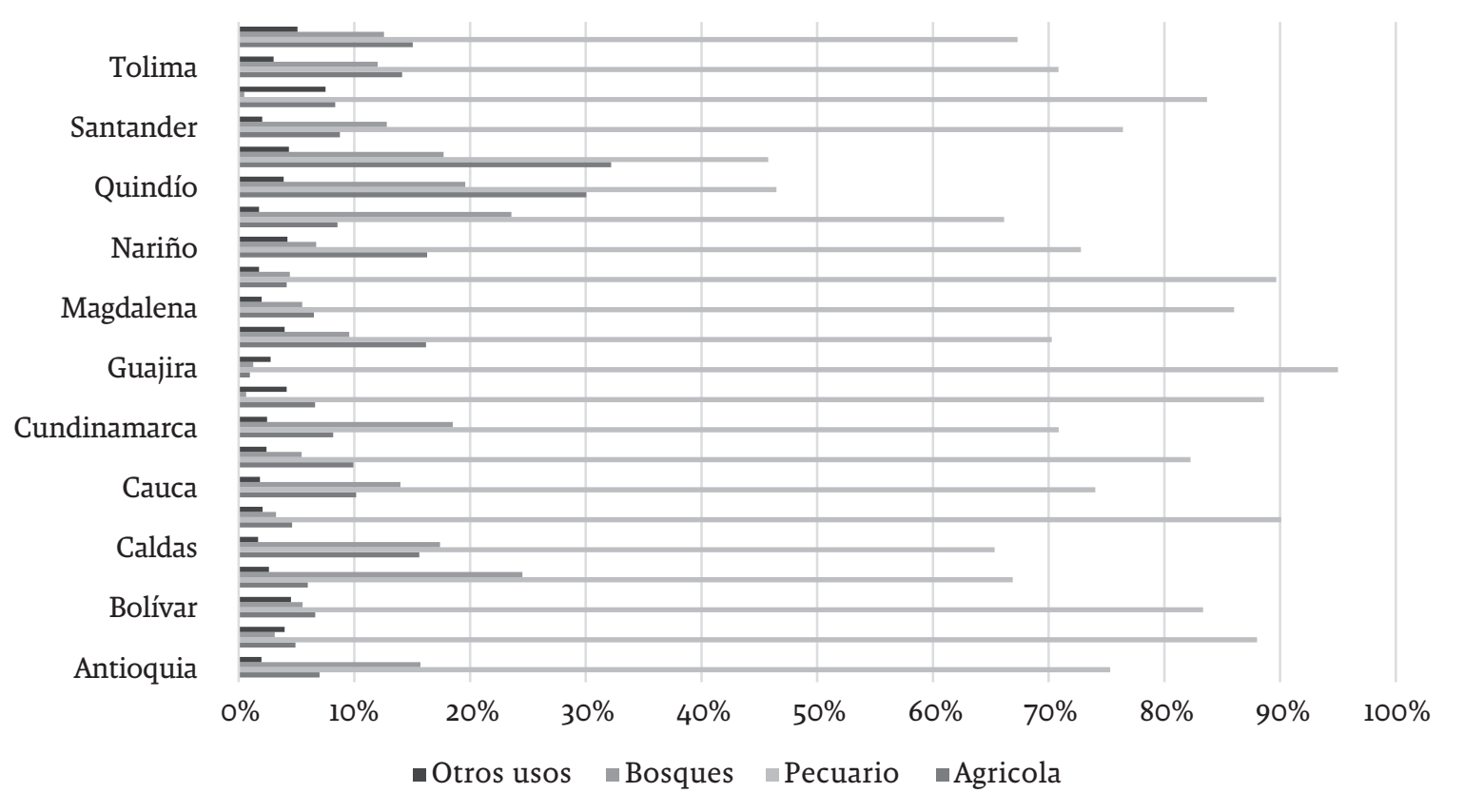

Fuente: elaboración propia con base en la ENA del DANE (2012b).

Figura 4. Participación de las empresas en las actividades económicas (2012)

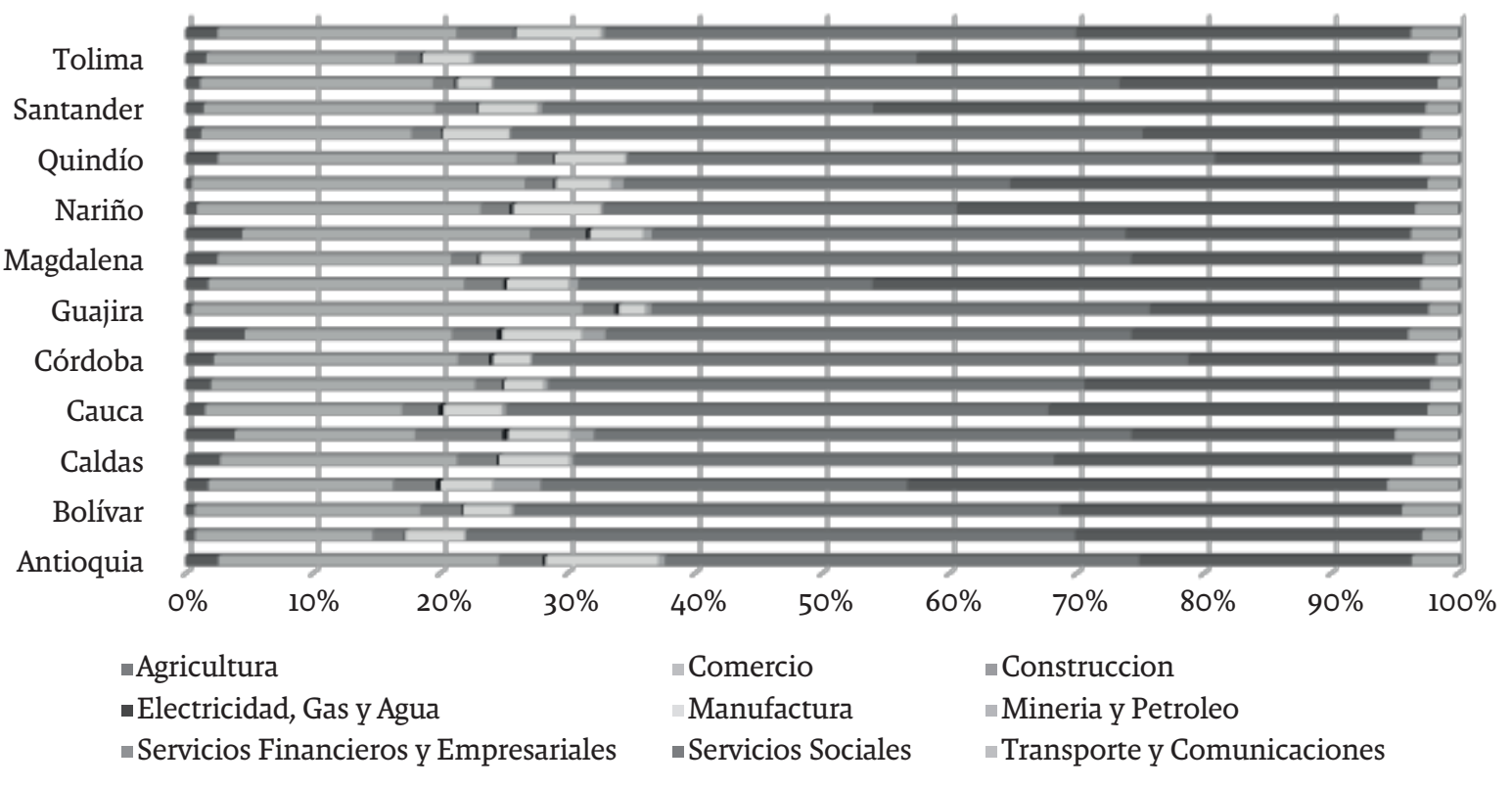

Fuente: elaboración propia con base en datos de BANCOLDEX (2012). 
En cuanto a las actividades de electricidad, Gas y Agua, en ninguno de los departamentos la participación empresarial alcanza el $1 \%$, siendo Casanare, Cauca y Cundinamarca los que más empresas poseen en ese sector.

Dentro del sector manufacturero, los departamentos con más empresas son los de Antioquia, Nariño y Valle del Cauca, respectivamente; dentro del mismo sector, los que menos participación empresarial poseen son los departamentos de Guajira, Sucre y Córdoba. Por otro lado, Boyacá, Casanare y Cundinamarca son los que más empresas tienen dentro de las actividades mineras y petroleras; $y$, los que menos tienen, son los departamentos de Risaralda, Córdoba y Quindío, respectivamente.

Los departamentos con menos participación empresarial en el sector financiero son los de Huila, Santander y Nariño; y los que cuentan con un mayor número de empresas, en este sector, son los departamentos de Córdoba, Risaralda y Sucre. Por su parte, Santander, Huila y Tolima tienen la concentración más alta de empresas del sector de servicios sociales, siendo Quindío, Córdoba y Casanare los que menos concentración muestran. Por otro lado, dentro del sector del transporte destacan los departamentos de Boyacá, Casanare y Bolívar, con el mayor número de empresas que participan en esta actividad; paralelamente, la menor cantidad se registra en los departamentos de Sucre, Córdoba y Cesar.

\subsection{Resultados de los indicadores que componen el índice de resiliencia}

Los datos anteriores permiten apreciar el contexto económico en el que se desenvolvían los 22 departamentos de la muestra en el año 2012. A continuación, se presentan los resultados del cálculo para los distintos indicadores que conforman el índice compuesto de resiliencia, seguido por los valores que se obtuvieron del cálculo del índice.

\subsubsection{Diversidad de suelos}

La Figura 5 muestra los resultados del índice de diversidad de suelos, el cual se calculó usando los datos de uso del suelo y aplicando el índice de Shannon; entre más cercanos estén los resultados a 2, quiere decir que existe mayor diversidad. Los valores así obtenidos expresan qué tanta heterogeneidad existe en el uso del suelo y en las actividades que se realizan en estos.

Los departamentos con mayor diversidad en el uso de los suelos son Risaralda y Quindío. La mayoría de los departamentos del interior del país presentan una diversidad de suelos que se encuentra en los intervalos de alta y media, mientras que los departamentos de la costa reflejan una baja diversidad. Los departamentos con la diversidad más baja son los de Guajira, Casanare, Meta y Córdoba.

\subsubsection{Diversidad de empresas}

Este índice es realizado de forma similar al anterior y refleja el nivel de diversificación de las actividades económicas existentes, dado el número de empresas totales que tiene cada departamento. Para su obtención, se usaron los datos de la participación empresarial y se aplicó la metodología de Shannon para capturar la diversidad específica. Entre más cercanos se encuentren los resultados a 3, quiere decir que existe mayor diversificación en las actividades empresariales que se realizan en los departamentos.

La Figura 6 refleja que los departamentos con una diversidad de empresas muy alta son los de Casanare, Cundinamarca, Boyacá, Meta, Antioquia, Valle y Caldas. A su vez, los departamentos que presentan la diversidad de empresas más baja son los de Sucre, Córdoba y Atlántico, respectivamente. El resto de departamentos se ubica mayoritariamente en el rango alto y medio, con excepción de Magdalena, Tolima, Guajira y Risaralda, que completan la lista de departamentos con una diversidad de empresas baja.

\subsection{3 Índice de desempeño integral}

El indicador de desempeño integral evalúa el desempeño de las entidades territoriales en cuanto a la eficacia en el cumplimiento de las metas de sus planes de desarrollo, su capacidad de gobierno y el desempeño fiscal, entre otros. Esta variable es utilizada como una aproximación del capital social en los departamentos, ya 
que, como se ha explicado anteriormente, los gobiernos elegidos son un reflejo del capital social de su población. Lo anterior se debe al hecho de que sociedades con altos niveles de estudio, de participación ciudadana, de sentido de pertenencia, son sociedades con una mayor formación de capital social, lo cual se refleja en la calidad de sus instituciones.

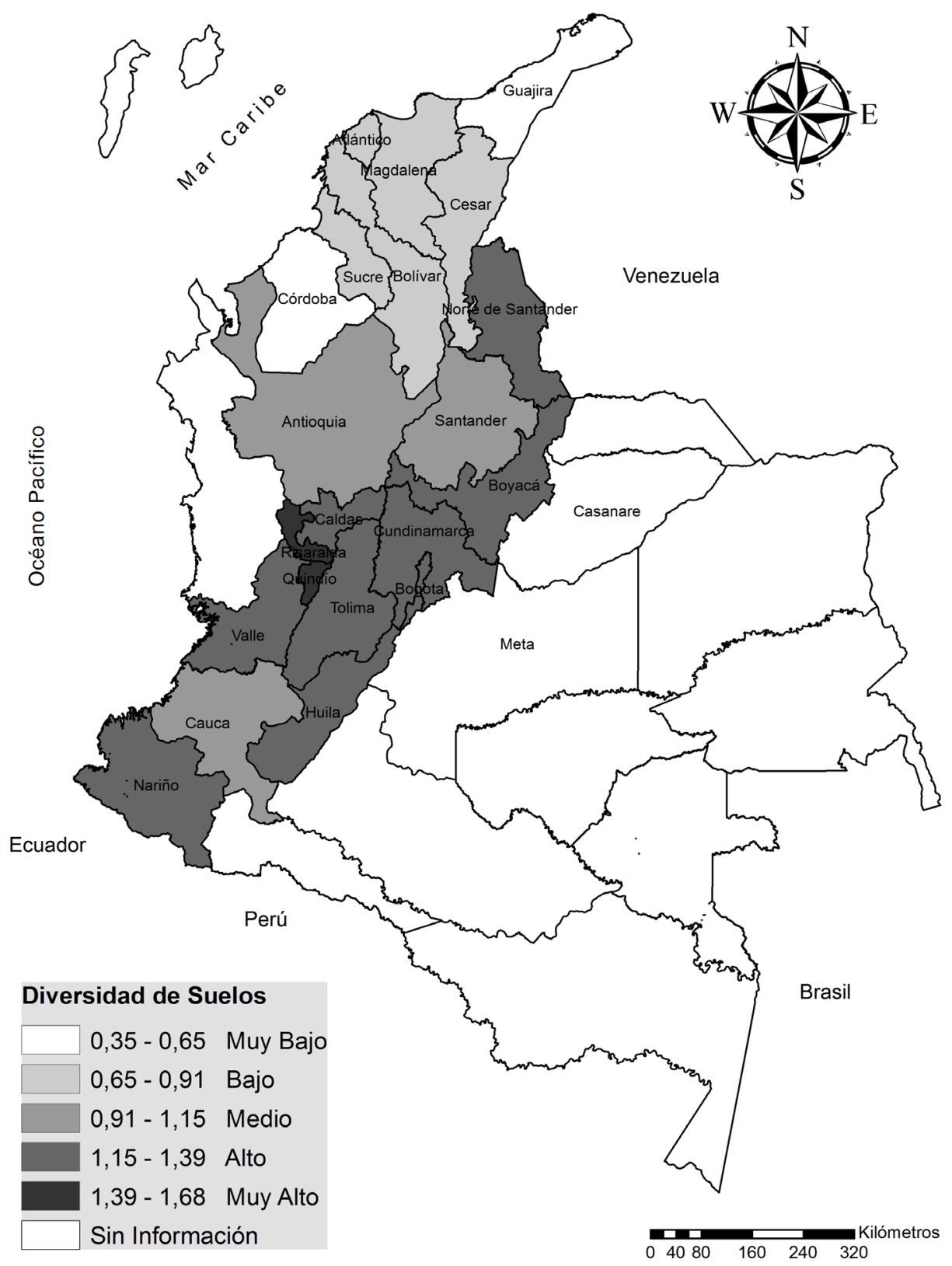

Fuente: elaboración propia con base en datos de la ENA del DANE (2012b). 


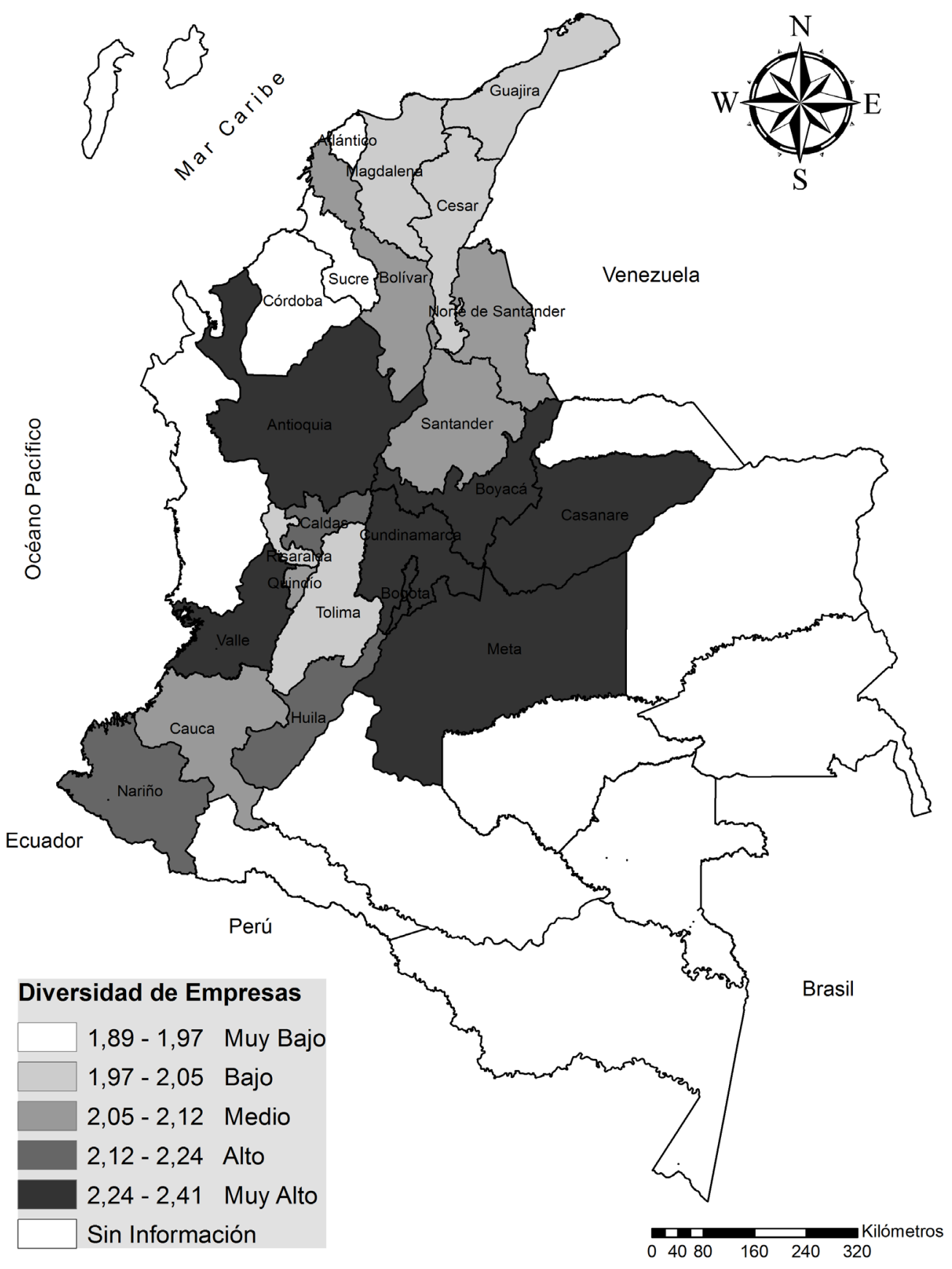

Fuente: elaboración propia con base en datos de BANCOLDEX (2012). 
Este indicador permite medir qué tan bueno ha sido un gobierno en el desarrollo de sus obligaciones y representa la dimensión social, que tiene un peso tan importante en este índice de resiliencia. $\mathrm{La}$ Figura 7 permite apreciar que los departamentos con mejor desempeño integral son los de Cundinamarca, Risaralda, Quindío y Guajira, respectivamente; y los de peor desempeño integral son Bolívar y Córdoba. La mayoría, entre los otros departamentos, se encuentra en los rangos de valoración media y alta.

Cabe resaltar que ningún departamento tuvo resultados muy buenos en términos reales. Esto teniendo en cuenta que el mayor promedio no llega a ocho, lo que es algo preocupante porque, en cierta medida, indica los problemas que tiene Colombia en términos de gobierno y se ve reflejado en la poca participación electoral de los ciudadanos. Esto, a su vez, nos indica un problema grave en términos de capital social.

\subsubsection{Huella Ecológica}

La huella ecológica es una variable usada para medir el impacto de las actividades humanas en el entorno en el que se desenvuelve. La Figura 8 y la Figura 9 muestran que los departamentos que presentan la huella ecológica más baja son los de Valle, Risaralda y Cundinamarca. Esto quiere decir que, a pesar de las actividades económicas que realizan, cada persona necesita entre 0,30 y 0,37 hectáreas para vivir.

La mayoría de departamentos presentan resultados dentro de la media del país. Departamentos como Atlántico, Quindío, Nariño, Caldas, Antioquia y Bolívar se mantienen con una huella ecológica baja, pues sus habitantes requieren menos de una hectárea por persona para demandar recursos y emitir residuos a los ecosistemas. El resto de departamentos, que corresponde al $60 \%$ de los considerados en este estudio, tienen una huella ecológica alta o muy alta, ya que requieren más de una hectárea por persona. En particular, los departamentos de Meta y Casanare reflejaron resultados muy por encima de la media. Esto debido a la poca población que poseen y la gran cantidad de tierra destinada a actividades pecuarias y agrícolas, factores que pesaron mucho al momento de determinar la huella ecológica (que, como se ha dicho, mide el consumo y el uso de los recursos naturales). A diferencia de los demás indicadores que componen el índice de resiliencia, en el caso de aquellos que representan la dimensión ambiental, es deseable que estos sean lo más bajo posible, ya que demuestra que las personas no necesitan de muchos recursos del entorno para llevar a cabo sus actividades.

\subsection{5 Índice compuesto de resiliencia}

La Figura 10 y la Figura 11 reflejan los resultados del índice compuesto de resiliencia que ha sido calculado usando las variables normalizadas de la diversidad de empresas, de suelos, el índice de desempeño integral, multiplicados por los ponderadores dados teóricamente a cada dimensión, y divididos entre el indicador de la huella ecológica sobre la superficie de cada departamento. Los resultados muestran que los departamentos con mayor resiliencia son Cundinamarca, Antioquia y Valle, respectivamente. Estos tres departamentos presentan una resiliencia muy alta, explicada por los muy buenos resultados de las dimensiones que conforman el índice.

En cuanto a la dimensión económica, representada por la diversidad de empresas y la diversidad de suelos, estos departamentos, a excepción de Antioquia, se mantuvieron entre los rangos alto y muy alto. Este resultado sugiere que, en caso de darse la recesión de un sector económico que afecte un determinado número de actividades, la diversidad económica ayudara a que no se estanque el departamento y pueda ser capaz de apoyarse en otras actividades, en respuesta a la crisis económica.

En cuanto al índice de desempeño integral que representa la dimensión social, Cundinamarca, Antioquia y Valle se mantienen entre los rangos alto y muy alto. Esto es señal de que sus gobiernos están cumpliendo con sus obligaciones o haciendo las cosas "bien", lo que también muestra altos niveles de capital social. Por ende, se espera que su capacidad de respuesta, al enfrentar un shock externo de cualquier tipo, sea rápida y eficaz, lo que a su vez ayudaría a minimizar los efectos del estrés y volver de forma acelerada a su punto de equilibrio. Algo similar sucede con la dimensión ambiental, en la que los tres departamentos presentaron buenos resultados (dado su bajo nivel de huella ecológica). Concretamente, y no obstante la densidad poblacional de estos territorios, cada habitante, en el desarrollo de sus actividades económicas, genera una carga sobre el ecosistema que no alcanza una hectárea. 


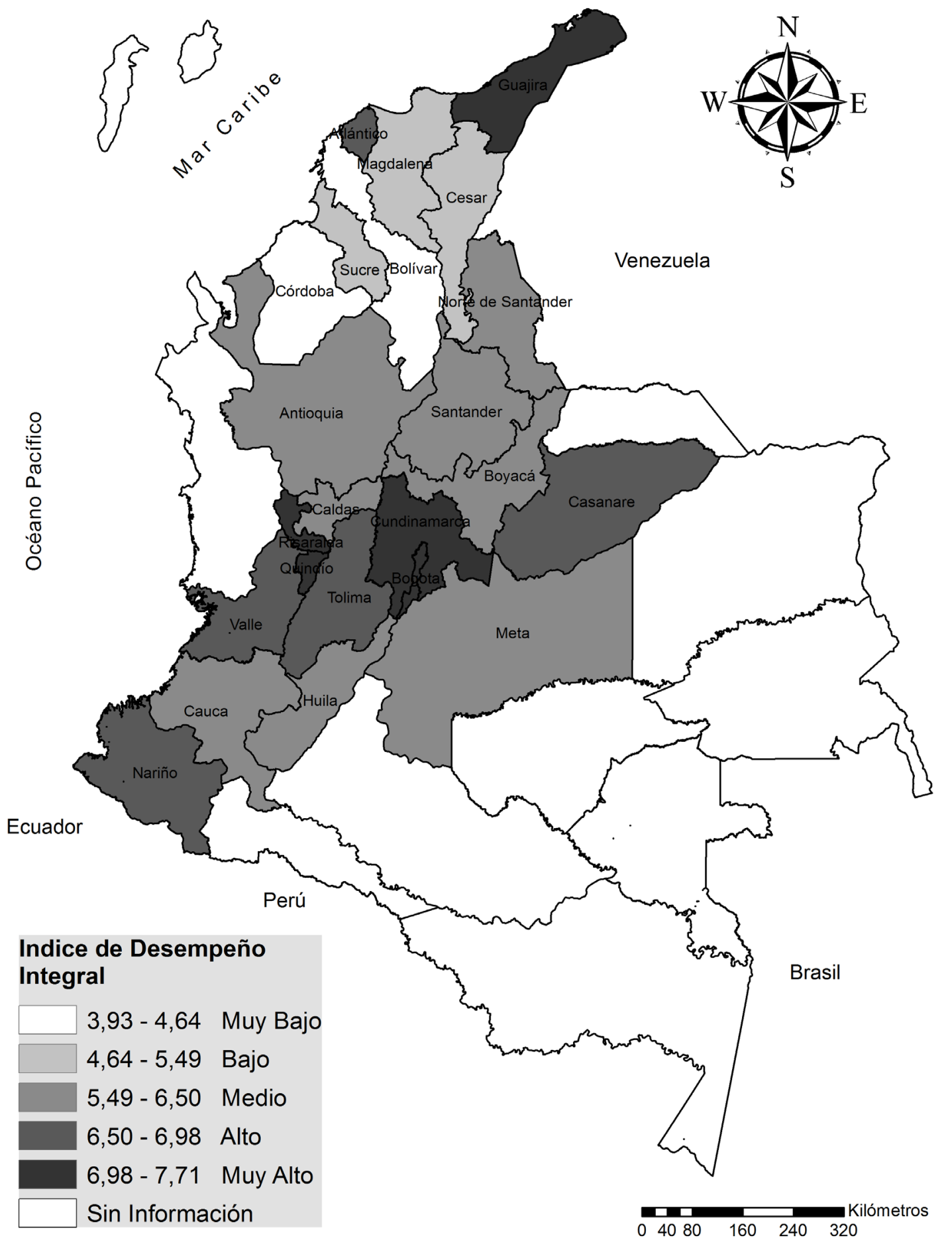

Fuente: elaboración propia con base en datos del panel municipal del CEDE (2012). 


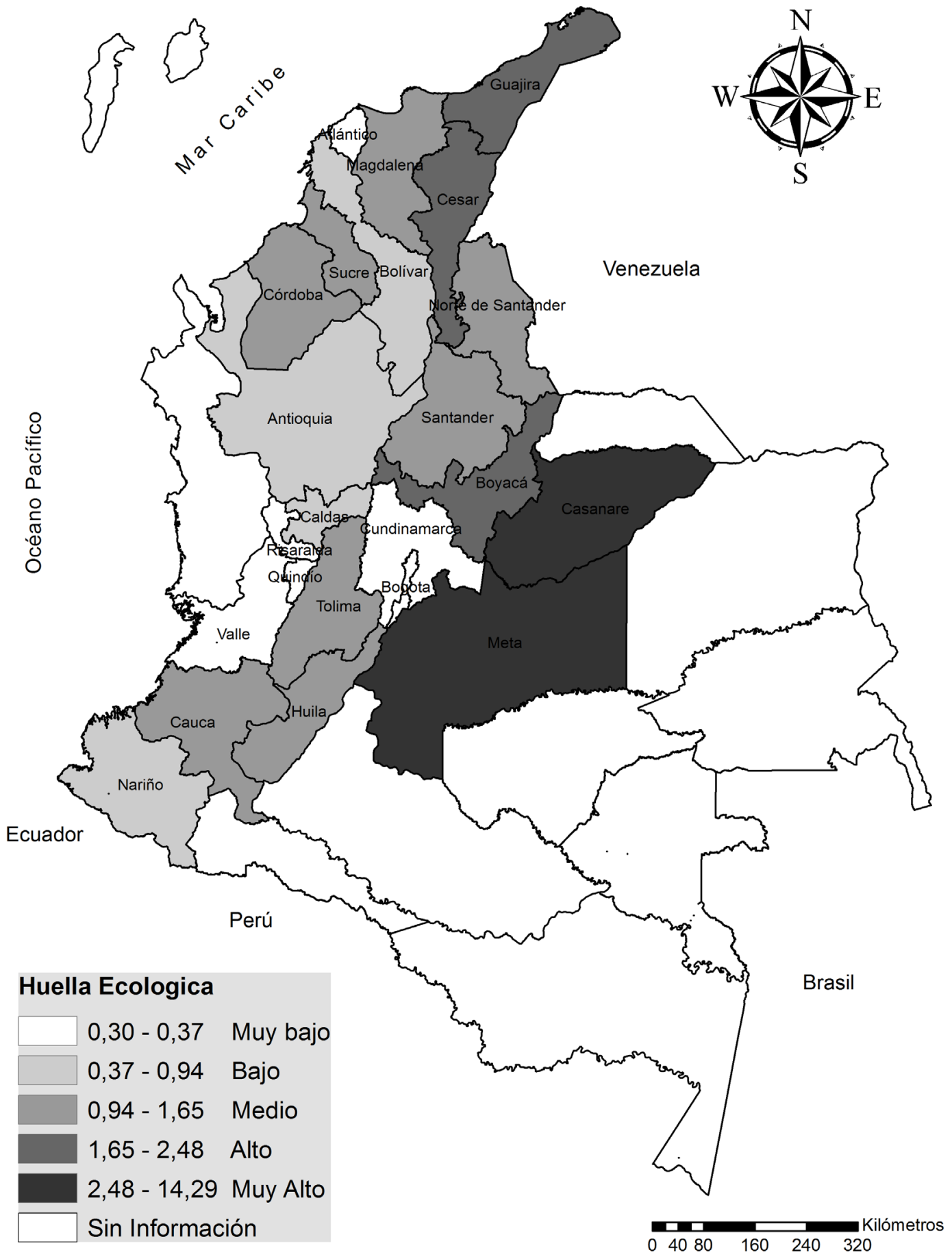

Nota: los datos se encuentran en hectáreas por personas.

Fuente: elaboración propia con base en datos del IDEAM (2012) y la ENA del DANE (2012b). 


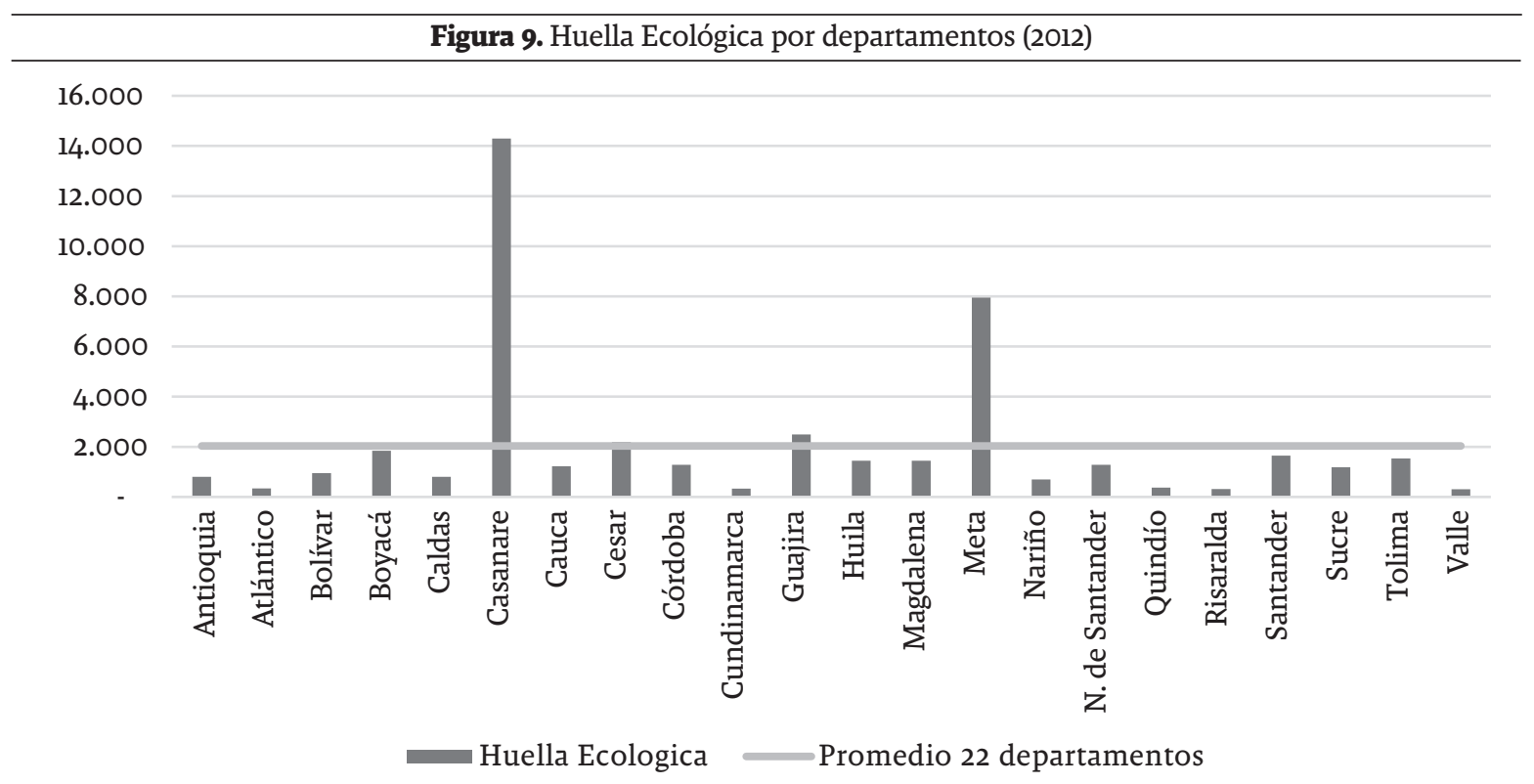

Nota: los datos se presentan en hectáreas por personas.

Fuente: elaboración propia con base en datos del IDEAM (2012) y la ENA del DANE (2012b).

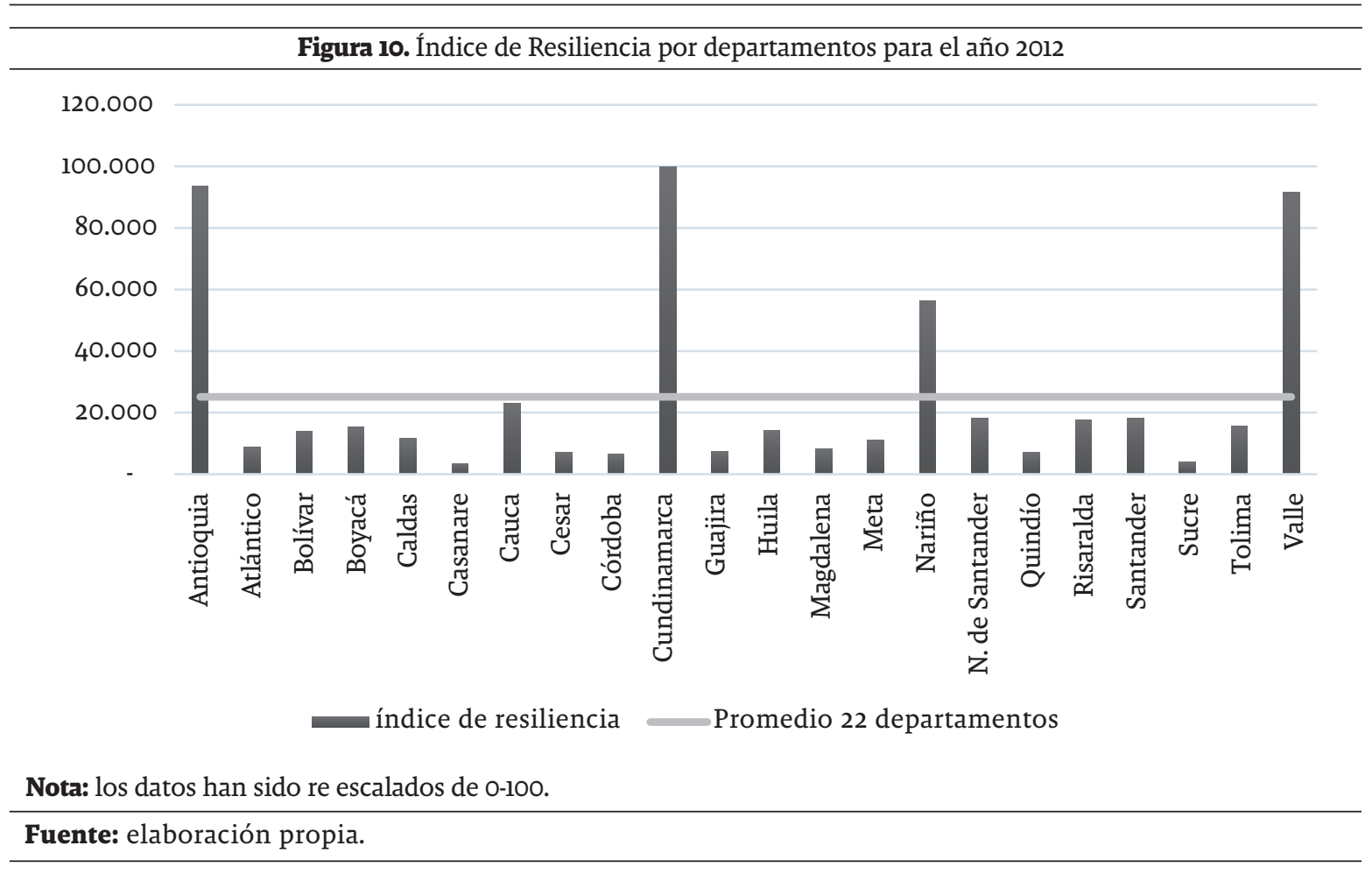




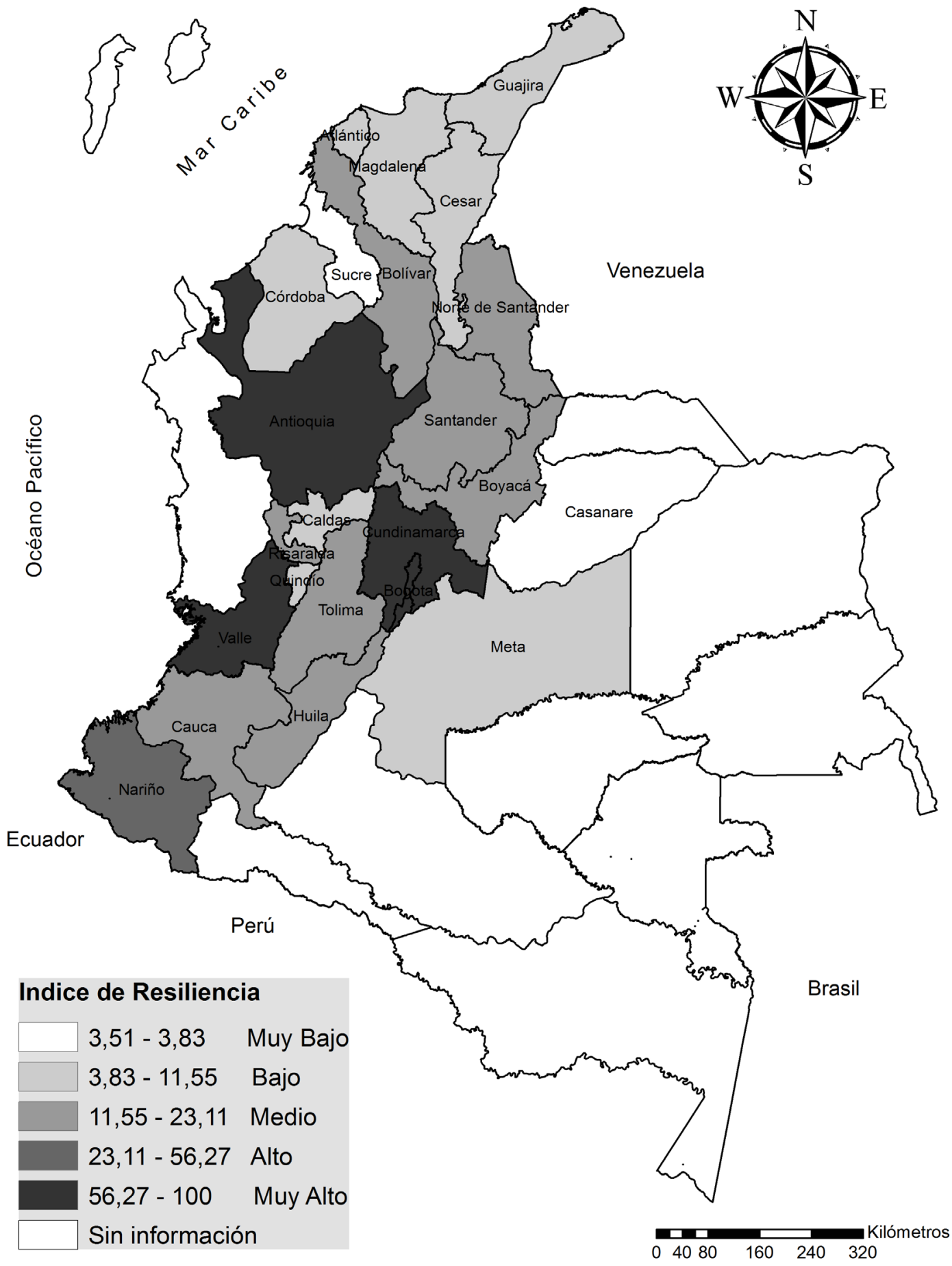

Nota: los valores del índice han sido re escalados para que tomen valores de 0-100.

Fuente: elaboración propia. 
Los sobresalientes resultados obtenidos por los tres entes territoriales, en cada una de las dimensiones que conforman el índice compuesto, explican su mayor resiliencia frente a los departamentos restantes, y es consecuente con su notable participación del $50 \%$ en la generación del PIB de Colombia.

Entre los 22 departamentos, también destacan los resultados de Nariño, ubicado por encima de la media y que, al igual que los tres anteriormente analizados, obtuvo un muy buen desempeño en las dimensiones que componen el índice. De esta manera, se ubica como el cuarto departamento con mejor resiliencia de la muestra estudiada.

Dicho lo anterior, se observa una gran brecha entre los cuatro departamentos mencionados y el resto de departamentos de la muestra. Ninguno de ellos supera la media de resiliencia y los resultados de este indicador son muy bajos, lo cual es un indicio de los precarios resultados que se obtienen en el resto de las dimensiones que componen el índice de resiliencia. Aunque estos departamentos alcanzaron un gran desempeño en algunas de las variables estudiadas, como ya se ha mencionado, la resiliencia es un concepto multidimensional y no depende solo de una esfera, sino del conjunto. Es decir, se trata de un índice holístico y, en tanto el territorio obtenga resultados sobresalientes en las dimensiones de interés, mayor será la probabilidad de que el sistema sea resiliente.

Los departamentos que tienen los peores resultados para el índice compuesto de resiliencia son Casanare, Sucre y Córdoba, respectivamente. Los departamentos de Sucre y Córdoba presentaron resultados muy bajos en todas las variables que conforman las tres dimensiones de estudio; por su parte, el departamento de Casanare tuvo un gran desempeño en cuanto a diversidad de empresas, pero los resultados en diversidad del suelo, el indicador de desempeño integral y la huella ecológica fueron muy bajos. Esto demuestra que la fortaleza en una dimensión no es suficiente para que un departamento se pueda considerar resiliente ya que, como se ha explicado, hace falta una buena coordinación con las demás categorías.

\section{Discusión de resultados}

Los resultados obtenidos en el cálculo del índice de resiliencia son consistentes con los de otros trabajos realizados sobre el tema, y se orientan de manera semejante. Por ejemplo, Suarez-Casado (2012), en la construcción del índice de resiliencia para las capitales de las provincias españolas, encontró que aquellas ciudades con altas huellas ecológicas, y que sobrepasan su capacidad de carga, son las menos resilientes. Del mismo modo, al comparar los resultados obtenidos por Rizzi et al. (2018), se concluye que las regiones europeas con mejores resultados en las dimensiones económica, social y ambiental son más resilientes, ya que poseen mejores impulsores de la resiliencia. Tal es el caso de la región escandinava. En el mismo sentido, los resultados expuestos por Vincent (2007), en su mayoría, se encuentran soportados en el bienestar económico y la estabilidad institucional. En general, estas ideas son respaldadas por Brooks et al. (2005), quienes encontraron que los países más vulnerables son aquellos con malos resultados en la categoría económica, social, ambiental y tecnológica.

En particular, sobre el trabajo que nos ocupa, un resultado importante a tomar en cuenta es la disparidad que mostraron los resultados del índice de resiliencia, pues solo tres departamentos Cundinamarca, Antioquia y Valle- presentaron una resiliencia muy alta, seguidos de lejos por Nariño; lo que nos indica que, aproximadamente, el $82 \%$ de los departamentos estudiados tendrían muchos problemas cuando se enfrenten a shocks externos, ya sean crisis económicas, políticas, desastres naturales, pandemias, etc. Este tipo de desigualdades son similares a las encontradas por Cuadrado-Roura y Maroto (2016), pues los desbalances evidenciados en la resiliencia de las regiones de España, desde un punto de vista de la dimensión económica, se deben a que los territorios más resilientes son aquellos que han tenido una alta especialización en actividades dinámicas y altamente productivas como la energía, algunas manufacturas y algunos servicios avanzados de mercado. 
Para el caso colombiano, algunos trabajos como los de Ramírez et al. (2007), Ramírez et al. (2014), y Vásquez-Bedoya et al. (2014) muestran que Cundinamarca, Antioquia y Valle son territorios pro cíclicos, además de ser las economías más diversificadas y tener mayor participación en el PIB nacional. De igual forma, esos departamentos presentan los mayores niveles de competitividad, productividad, fortaleza económica y capital humano, condiciones esenciales que ayudan a incrementar los niveles de resiliencia en los territorios.

Otro resultado destacable es la importancia de la diversidad. Al respecto, Walker y Salt (2006), y Hopkins (2008) han señalado que la mayor diversidad hace a los sistemas más resilientes. Esto debido a que, en un socio-ecosistema, la presencia de grupos homogéneos los hace más vulnerables ante un estrés del entorno. Esto mismo sucede en los entes territoriales con economías poco diversificadas, pues la homogeneidad en sus actividades económicas y empresariales las hace proclives a tener una menor capacidad de adaptación ante un evento traumático. Un aspecto también importante es la relación entre resiliencia y sostenibilidad, pues nuevas corrientes de estudio, sobre indicadores sostenibles, expresan que para que un sistema sea sostenible este debe ser resiliente, tal como lo explican Tumini (2016), Perrings (2006), CamposGarcía (2013) y Martin-Palmero et al. (2004).

Como se ha evidenciado, los investigadores han dado gran importancia al abordaje del tema de la resiliencia territorial y la necesidad de que este sea tenido en cuenta por los tomadores de decisiones. Todos los territorios están expuestos a sufrir algún evento traumático y la capacidad de recuperación que estos muestren es fundamental para el bienestar de las personas que los habitan.

\section{Conclusiones}

La capacidad de resiliencia de un territorio le permite, ante una eventualidad negativa, pasar de un estado de equilibrio a otro sin que la transición sea muy traumática. La resiliencia es un concepto multidimensional, el cual involucra todas las esferas que componen la realidad de un territorio. En efecto, para la construcción del índice compuesto de resiliencia, se usaron variables que representan las categorías económica, social y ambiental de los departamentos en cuestión.

Los resultados reflejan que dentro del ámbito económico de un territorio es preferible que exista heterogeneidad en las actividades económicas que se realizan, pues de esta forma se garantiza que, ante una coyuntura que afecte a un sector, el resto de actividades pueda seguir desarrollándose. Con respecto a la dimensión social, se hace énfasis en la importancia de tener altos niveles de capital social, puesto que este impacta directamente a la hora de tener instituciones eficientes que sean capaces de responder, de manera rápida y efectiva, ante cualquier eventualidad. Con relación a la dimensión ambiental, toma vital importancia el impacto que tienen las actividades humanas en el medio ambiente. En ese sentido, un departamento será más resiliente en tanto sea más balanceado en la dimensión económica, social y ambiental.

Los departamentos que presentaron los mejores resultados, en el índice de resiliencia, fueron Cundinamarca, Antioquia y Valle del Cauca, caracterizados por ser motores de la economía colombiana, sin dejar de lado el hecho de que presentan altos niveles de capital social representados en su sentido de pertenencia, sus altos niveles educativos, participación en las urnas y en la eficiencia mostrada por sus instituciones. Todo esto sin descuidar el impacto que tiene el desarrollo de sus actividades en el medio ambiente.

En el lado opuesto, se encuentran Casanare, Sucre y Córdoba, cuyo rendimiento fue de los más bajos de entre los departamentos analizados. Para el caso de Casanare se evidenciaron resultados favorables en su diversidad empresarial, sin embargo, este se vio empañado por el bajo desempeño en el resto de componentes del índice. Con respecto a Sucre y Córdoba, sus resultados fueron pobres en todas las dimensiones del índice, lo cual es un aspecto preocupante para la región Caribe colombiana.

Este trabajo permite observar que, todavía, son los territorios centrales los que condensan 
la mayoría del desarrollo económico, político y social en el país, lo cual debe equilibrarse para impulsar un verdadero crecimiento integral de Colombia. Esto implica trabajar en la disminución de las grandes diferencias entre la capacidad de resiliencia de Cundinamarca, Antioquia y Valle del Cauca con el resto de departamentos del país. Cerrar estas brechas pasa por ser capaces de tener resultados más equilibrados en el ámbito económico, social y ambiental, lo cual se verá reflejado en un aumento en la calidad de vida de todos los habitantes y, por ende, mejores resultados a nivel país.
Por último, es importante resaltar la conveniencia de que los hacedores de políticas y los tomadores de decisiones evalúen con atención los resultados de los estudios sobre resiliencia territorial. En épocas tan complejas en términos sociales, políticos, económicos y ambientales, en las que se encuentra inmerso el mundo en general, los eventos adversos tienen una alta probabilidad de ocurrencia. Por lo tanto, la disponibilidad de una buena capacidad de respuesta es una herramienta fundamental para la sostenibilidad de los territorios en el mediano y largo plazo.

\section{Referencias}

Adgner, W. (2000). Social and ecological resilience: are they related? Progress in Human Geography, 24(3), 347-364. https://doi.org/10.1191/030913200701540465

BANCOLDEX -Banco de Comercio Exterior de Colombia-. (2012). Datos de sectores productivos (PILA). http://datlascolombia.com/\#/

Berga, L. (2017). Resiliencia territorial frente a las inundaciones. Revista de Obras Públicas: Órgano profesional de los ingenieros de caminos, canales y puertos, (3587), 74-81.

Berkes, F. y Ross, H. (2016). Panarchy and community resilience: Sustainability science and policy implications. Enviromental Science y Policy, 61, 185-193. https://doi.org/10.1016/j.envsci.2016.04.004

Brand, F. S. y Jax, K. (2007). Focusing the meaning(s) of resilience: Resilience as a descriptive concept and a boundary object. Ecology and Society, 12(1), 23.

Brooks, N., Adger, W. y Kelly, P. (2005). The determinants of vulnerability and adaptive capacity at the national level and the implications for adaptation. Global environmental change, 15(2), 151-163. https://doi.org/10.1016/j.gloenvcha.2004.12.006

Bruneau, M., Chang, S., Eguchi, R., Lee, G., O'Rourke, T., Reinhorn, A., ... Von Winterfeldt, D. (2003). A Framework to Quantitatively Assess and Enchance the Seismic Resilience of Communities. Earthquake Spectra, 19(4), 733-752. https://doi.org/10.1193/1.1623497

Campos-García, M. (2013). La Huella Ecológica del agua industrial en territorios insulares: sostenibilidad versus resiliencia. Universidad de Las Palmas de Gran Canaria.

Carpenter, S., Walker, B., Anderies, J. y Abel, N. (2001). From metaphor to measurement: resilience of what to what? Ecosystems, 4(8), 765-781. https://doi.org/10.1007/s10021-001-0045-9

CEDE -Centro de Estudios sobre Desarrollo Económico-. (2012). Catálogo de datos. https://datoscede. uniandes.edu.co/es/catalogo-de-microdata

CEPAL -Comisión Económica para América Latina y el Caribe-. (2018). Potenciar la resiliencia de las ciudades y sus territorios de pertenencia en el marco de los acuerdos sobre cambio climático y de la Nueva Agenda Urbana. CEPAL. 
Christopherson, S., Michie, J. y Tyler, P. (2010). Regional resilience: theoretical and empirical perspectives. Cambridge journal of regions, economy and society, 3(1), 3-10. https://doi.org/10.1093/ cjres/rsq004

Cuadrado-Roura, J. R. y Maroto, A. (2016). Unbalanced regional resilience to the economic crisis in Spain: a tale of specialisation and productivity. Cambridge Journal of Regions, Economy and Society, 9(1), 153-178. https://doi.org/10.1093/cjres/rsv034

DANE -Departamento Administrativo Nacional de Estadística-. (2012a). Cuentas nacionales departamentales. https://www.dane.gov.co/index.php/estadisticas-por-tema/cuentas-nacionales/ cuentas-nacionales-departamentales

DANE -Departamento Administrativo Nacional de Estadística-. (2012b). Encuesta nacional agropecuaria (ENA). https://www.dane.gov.co/index.php/estadisticas-por-tema/agropecuario/ encuesta-nacional-agropecuaria-ena/encuesta-nacional-agropecuaria-por-departamentos

Dynes, R. (2002). The importance of social capital in disaster response. Preliminary paper, (327), 1-59.

Folke, C., Carpenter, S., Walker, B., Scheffer, M., Chapin, T. y Rockstrom, J. (2010). Resilience thinking: Integrating resilience, adaptability and transformability. Ecology and society, 15(4), 20-28.

Foster, K. (2012). In search of regional resilience. En M. Weir, N. Pindus, H. Wial, y H. Wolman (Eds.), Urban and Regional Policy and its effects (pp. 24-59). Brookings Institution Press.

Gunderson, L. (2010). Ecological and human community resilience in response to natural disasters. Ecology and Society, 15(4), 1-11.

Hamdouch, A., Depret, M. y Tanguy, C. (2012). Mondialisation et résilience des territoires: Trajectoires, dynamiques d'acteurs et expériences. Presses de l'Université du Québec.

Hassink, R. (2010). Regional resilience: a promising concept to explain differences in regional economic adaptability? Cambridge Journal of Regions, Economy and Society, 3(1), 45-58. https://doi. org/10.1093/cjres/rspo33

Holling, C. S. (1973). Resilience and stability of ecological systems. Annual Review of Ecology and Systematics, 4(1), 1-23. https://doi.org/10.1146/annurev.es.04.110173.000245

Holling, C. S. (1996). Engineering resilience versus ecological resilience. En P. Schulze (Ed.), Engineering within ecological constraints (pp. 31-44). National Academy of Engineering.

Hopkins, R. (2008). The transition handbook. Green Books.

IDEAM -Instituto de Hidrología, Meteorología y Estudios Ambientales-. (2012). Inventario Nacional y Departamental de Gases Efecto Invernadero-Colombia. IDEAM.

Labandeira, X., Carmelo, L. y Vázquez, M. X. (2007). Economía Ambiental. Pearson Educación S.A.

Ledogar, R. J. y Fleming, J. (2008). Social capital and resilience: A review of concepts and selected literature relevant to aboriginal youth resilience research. Pimatisiwin, 6(2), 25-46.

Loh, J. y Wackernagel, M. (2004). Living planet report 2004. World Wide Fund for Nature.

Magis, K. (2010). Community resiience: An indicator of social sustainability. Society \& Natural Resources: An International Journal, 23(5), 401-416. https://doi.org/10.1080/08941920903305674 
Martin-Breen, P. y Anderies, J. M. (2011). Resilience: A literature review. The Bellagio Initiative.

Martin-Palmero, F., González-Laxe, F., Miguélez-Pose, F., Menéndez-Pérez, E. y Dopico-Castro, J. (2004). Formulación de los modelos de desarrollo sostenible. En F. Martin-Palmero (Ed.), Desarrollo sostenible y huella ecológica: una aplicación a la economía gallega (pp. 39-50). NETBIBLO.

Méndez, R. M. (2013). Estrategias de innovación para el desarrollo y la resiliencia de ciudades medias. Documents d'análisi geográfica, 59(3), 481-499. https://doi.org/10.5565/rev/dag.60

Méndez, R. (2015). Redes de colaboración y economía alternativa para la resiliencia urbana: una agenda de investigación. biblio $W$ revista bibliográfica de geografía y ciencias sociales, 21(1139), 1-24.

Milman, A. y Short, A. (2008). Incorporating resilience into sustainability indicators: An example for the urban water sector. Global Environmental Change, 18(4), 758-767. https://doi.org/10.1016/j. gloenvcha.2008.08.002

Norris, F., Stevens, S., Pfefferbaum, B., Wyche, K. y Pfefferbaum, R. (2008). Community resilience as a metaphor, theory, set of capacities, and strategy for disaster readiness. Am J Community Psychol., 41(2), 27-50. https://doi.org/10.1007/s10464-007-9156-6

Organización de las Naciones Unidas. (2005). Marco de Acción de Hyogo para 2005-2015: Aumento de la resiliencia de las naciones y las comunidades ante los desastres. Conferencia Mundial sobre la Reducción de los Desastres, Kōbe, Japón.

Perrings, C. (2006). Resilience and sustainable development. Environment and Development Economics, 31(5), 417-427. https://doi.org/10.1007/s10464-007-9156-6

Phillips, J., Duque, A., Scott, C., Peña, M., Franco, C., Galindo, G., ... Cárdenas, D. (2014). Aportes técnicos del Sistema de Monitoreo de Bosques y Carbono a la propuesta de preparación de Colombia para $R E D D+$ : datos de actividad y factores de emisión. IDEAM.

Pike, A., Dawley, S. y Tomaney, J. (2010). Resilience, adaptation and adaptability. Cambridge journal of regions, economy and society, 3(1), 59-70. https://doi.org/10.1093/cjres/rsqool

Pimm, S. (1984). The complexity and stability of ecosystems. Nature, 307(5949), 321-326. https://doi. org/10.1038/307321ao

Polèse, M. (2010). The resilient city: on the determinants of successful urban economies. University of Québec.

Putnam, R. (1993). Making Democracy Work: Civic Traditiopns in Modern Italy. Princeton University Press.

Quarantelli, E. (2004). Emergent behaviors and groups in the crisis time periods of disasters. Disaster Research Center University of Delaware, (206), 1-17.

Ramírez, J. C., Osorio, H. y Parra-Peña, R. I. (2007). Escalafón de la competitividad de los departamentos en colombia (Vol. 17). United Nations Publications.

Ramírez, J. C., Parra-Peña, R. I., González, L. y Corredor, A. (2014). Escalafón de la competitividad de los departamentos de Colombia, 2012-2013 (Vol. 27). United Nations Publications. 
Rizzi, P., Graziano, P. y Dallara, A. (2018). A capacity approach to territorial resilience: the case of European regions. The Annals of Regional Science, 60(2), 285-328. https://doi.org/10.1007/ s00168-017-0854-1

Rothstein, B. y Stolle, D. (2002). How political institucions create and destroy social capital: an institucional theory of generalized trust. American Political Science Association.

Rueda, S. (2011). El urbanismo ecológico: un nuevo urbanismo para abordar los retos de la sociedad actual. http://www.upv.es/contenidos/CAMUNISO/info/UrbanismoEcologicoSRueda.pdf

Sánchez-Zamora, P., Gallardo-Cobos, R. y Ceña-Delgado, F. (2014). El medio rural andaluz frente a la crisis económica: un análisis de los factores de resiliencia territorial. Economía Agraria y Recursos Naturales, 14(1), 27-56. https://doi.org/10.7201/earn.2014.01.02

Suarez-Casado, M. (2012). La resiliencia de los ecosistemas urbanos: una propuesta de evaluación para la sostenibilidad. Universidades Autónoma y Complutense de Madrid.

Tumini, I. (2016). Acercamiento teórico para la integración de los conceptos de Resiliencia en los indicadores de Sostenibilidad Urbana. Revista de Urbanismo, (34), 4-20. https://doi. org/10.5354/0717-5051.2016.40056

Vásquez-Bedoya, F., Restrepo-Ochoa, S. I., Lopera-Castaño, M. y Restrepo-Estrada, M. (2014). Los Ciclos Económicos Departamentales En Colombia, 1960-2011. Revista de economía institucional, 16(30), 271-285.

Vincent, K. (2007). Uncertainty in adaptive capacity and the importance of scale. Global Environmental Change, 17(1), 12-24. https://doi.org/10.1016/j.gloenvcha.2006.11.009

Wackernalgel, M. y Rees, W. (1996). Our Ecological Footprint: Reducing human impact on Earth (Vol. 9). New society publishers.

Walker, B. y Salt, D. (2006). Resilience thinking: sustaining ecosystems and people in a changing world. Island press.

Walker, B., Holling, C. S., Carpenter, S. y Kinzig, A. (2004). Resilience, adaptability and transformability in social-ecological systems. Ecology and Society, 9(2), 5.

Yohe, G. y Tol, R. S. (2002). Indicators for social and economic coping capacity-moving toward a working definition of adaptive capacity. Global Environmental Change, 12(1), 25-40. https://doi. org/10.1016/S0959-3780(01)00026-7 